\title{
Seizure commonly associated with Neurocysticercosis are not linked with pork meat diet. A retrospective analysis
}

\author{
Nisha Khanal', Reena Shrestha² \\ 1,2 Medical Officer, Department of Neuromedicine, College of Medical Science and Teaching Hospital, Bharatpur, Nepal
}

A B S TR A C T

Background: Neurocysticercosis (NCC) is a neglected tropical disease and common cause of epilepsy in developing countries. Aims and Objectives: The purpose of the study was to determine the prevalence of NCC and factor associated with the cause of NCC in Nepalese patients. Material and Methods: All patient with complain of seizure admitted to the College of Medical Sciences, and Teaching Hospital (COMS-TH), Nepal for a period of 2016 were included in the study. All age group patients between 10-80 years old with confirmed diagnosed of NCC were considered for the retrospective analysis. The proportion distribution of NCC was categorized based on the etiology of seizure, demographic profile (gender and age) and dietary habit of patients. Results: Among 142 patients with seizure, 54(38\%) were computerized tomography (CT) scan confirmed NCC cases in which 29 (54\%) were male, and $25(46 \%)$ were female. Almost $44(81.5 \%)$ patients with NCC were below the age of 50 years, and high prevalence $17(31.5 \%)$ was observed at the age of $10-20$ years old patients. All patients depend on the agricultural background, in which $35(65 \%)$ were farmer and $19(35 \%)$ with an agricultural background. Additionally, $10(18.5 \%)$ patients with NCC were vegetarian, 44 (81.5\%) were non-vegetarian. Among non-vegetarian, only 2 (4.5\%) were pork eaters. Most of the patients had poor hygienic habits and eating raw vegetables. All patients were managed with the single antiepileptic drug but, Albendazole plus steroid therapy was added in patients with active lesion observed in CT scan. Conclusions: This study showed a high prevalence of NCC and a common cause of the new onset of seizures in the patient of COMS-TH, Nepal. Also, our data showed that NCC is not associated with pork eaters because NCC was observed more number in vegetarian compared to patients who consume pork meat diet. NCC in patients with seizure was solely due to poor personal hygiene.

Key words: Neurocysticercosis; Seizure; Taenia solium; Pork Eater; Nepal

\section{INTRODUCTION}

Neurocysticercosis (NCC) is a serious neuroparasitic disease worldwide and common identifiable cause of new onset seizure in south Asia including Nepal. However, a preventable parasitic infection of the brain parenchyma caused by the ingestion of the eggs of the tapeworm Taenia solium. ${ }^{1}$ Infection with T. solium (Taeniasis) occurs in both pigs and human, in which both act as an intermediate host for the larval form called cysticercus, and human is the definite host for the adult tapeworm. ${ }^{2}$ The transmission of NCC involves consumption of contaminated foods or water with an eggs of T. solium, which occurs in cyclic fashion between pork and human that initially infect the intestine. This intestinal parasite enters systemic circulation by penetrating gut wall and lodge in central nervous system causing NCC. Also, NCC is endemic in many low-and middle-income countries (LMICs) with poverty-stricken areas of the world ${ }^{3}$ and listed as neglected tropical disease on the world health organization (WHO). ${ }^{4}$ The prevalence of NCC in patients with seizure in mean age group of $33.9 \pm 16.4$ was $16 \%$ (21 out of 131). ${ }^{5}$ However, in children 
inconsistent prevalence of NCC was observed between $16.9 \%(229 \text { out of } 1355)^{6}$ and $43 \%$ (72 out of 168$) .^{7}$ In the present study, we reported the prevalence of NCC in seizure patients and association of NCC with dietary habits, in which pork eater are not directly linked.

\section{MATERIALS AND METHODS}

The present study was conducted at the College of Medical Sciences and Teaching Hospital (COMS-TH), Bharatpur, Nepal. Study approval was obtained from the COMSTH research committee and performed a retrospective analysis. All seizure cases that were diagnosed and treated in Neuromedicine ward of COMS-TH for a year in 2016 was included. A total of 142 patients with complain of seizure disorders were admitted based on the record maintained in the hospital. Among 142 cases, 54 seizure cases were associated with Neurocysticercosis (NCC) and observed in all age group, including children, adult, and old patients. Further, NCC diagnosis was based on the COMS-TH diagnostic criteria, such as Cystecercal serology positive, neuroimaging findings, and histopathology of the subcutaneous nodule.

All patients with at least one episode of seizure were included, while newly diagnosed post cerebrovascular accident (CVA) were excluded from the study. In the present study, cases diagnosed with NCC were analyzed for their dietary habits (vegetarian, non-vegetarian) and the standard of their hygiene. Also, non-vegetarian diets were categorized as pork eaters (PE) and general meat consumers (GMC).

\section{RESULTS}

High prevalence of Neurocysticercosis (NCC) in Seizure The etiology of seizure was categorized as shown in Figure 1 based on the patients complain, clinical symptoms, and diagnosis. Out of 142 cases of seizure complain, $38 \%$ $(n=54)$ were associated with NCC, followed by $21 \%(n=30)$ with unknown etiology, $11 \%(n=10)$ with post CVA, and $10 \%(n=14)$ with alcohol withdrawal seizure. Other etiology with less prevalent includes, $6 \%(n=8)$ of acute encephalitic syndrome (AES), $5 \%(n=7)$ of atrophy/hemiatrophy, $4 \%$ $(n=5)$ of post-traumatic, $2 \%(n=3)$ of psychogenic nonencephalitic seizure, and $4 \%(n=5)$ of rare causes (like hyponatremia, hemangioma brain, postpartum, cortical dysplasia, and Fahr's syndrome). Our data revealed that NCC is the more prevalent and common cause of the seizure.

\section{Categorization of NCC based on the demographic profile}

The seizure patients diagnosed with NCC $(n=54)$ were further categorized based on gender and age group. Our study showed that NCC was observed more in male of $54 \%$ $(n=29)$ compared to female of $46 \%(n=25)$, as shown in Figure $2 \mathrm{a}$. In addition, the distribution of NCC-dependent seizure was grouped based on the age of the patients. The high prevalence of NCC was found in the age group of 10-20, while less prevalence in 30-40 as shown in Figure $2 \mathrm{~b}$. Of the total patients, $31.5 \%(n=17)$ in $10-20$ years, $22 \%$ $(n=12)$ in $20-30$ years, $13 \%(n=7)$ in $30-40$ years, $15 \%$ $(n=8)$ in $40-50$ years, and $18.5 \%(n=10)$ in $>50$ years of age group. Together, our data showed a high prevalence of NCC in 10-20 years of age group, and significant gender bias was observed.

Dietary and personal hygiene correlation with NCC To know the cause of NCC, dietary habit (vegetarian and non-vegetarian) was correlated with the patients presenting NCC $(n=54)$. Based on dietary habit, $18.5 \%(n=10)$ of vegetarian and $81.5 \%(n=44)$ non-vegetarian (both $\mathrm{PE}$ and GMC) patients were diagnosed with NCC and seizure as shown in Figure 3 a. But, out of 44 non-vegetarians, only $4.5 \%(n=2)$ were pork eaters (PE) and $95.5 \%(n=42)$

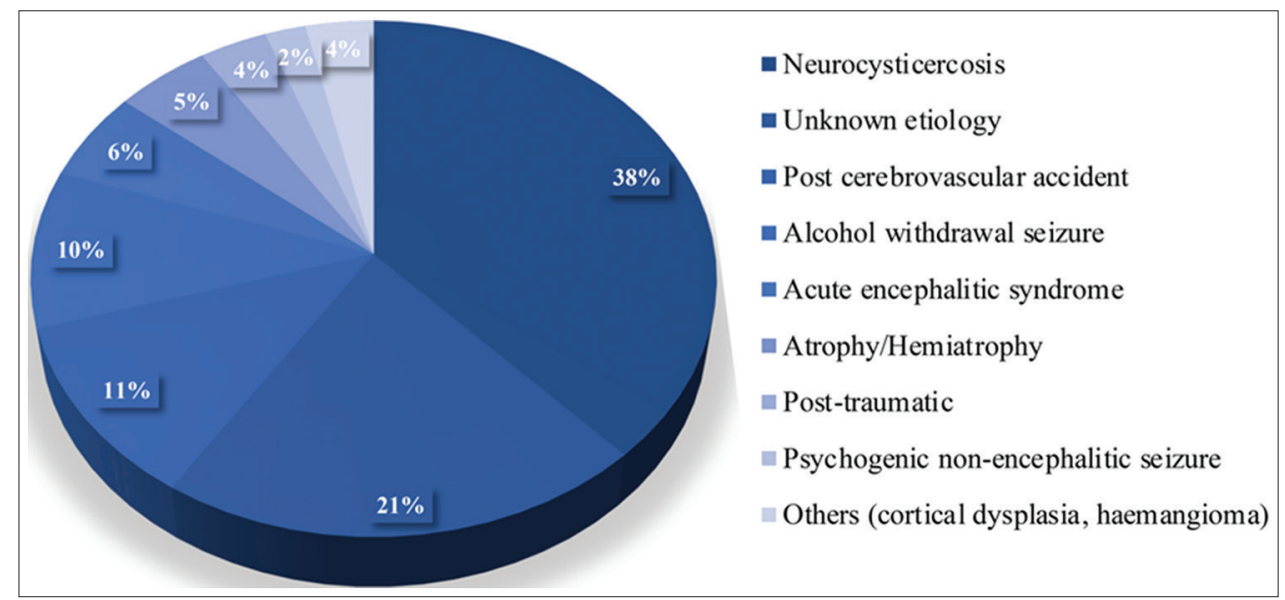

Figure 1: Representative pie chart showing various etiology of seizure and percentage prevalence 


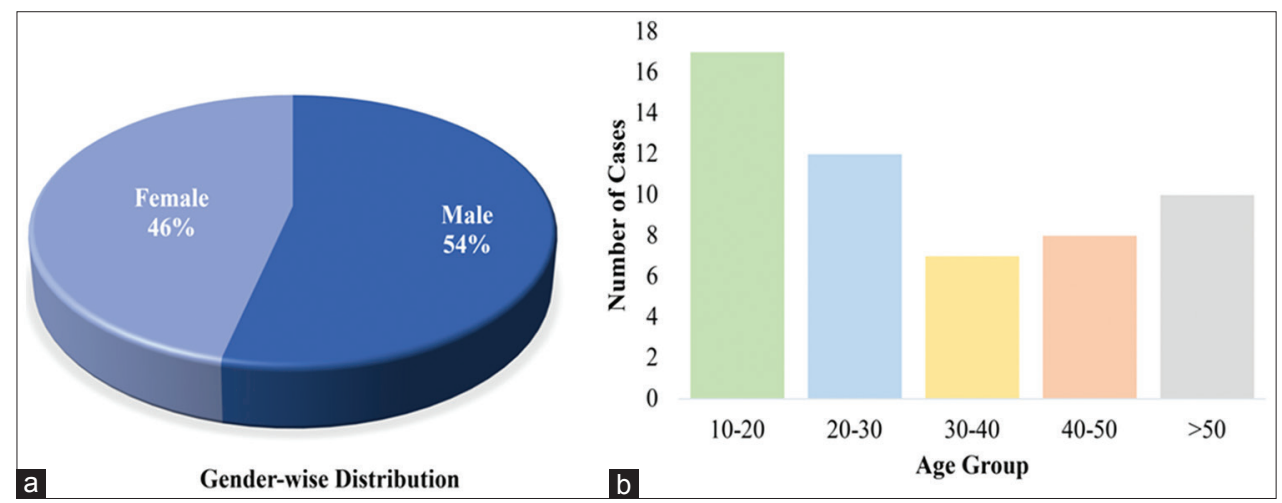

Figure 2: Demographic distribution for NCC in seizure patients. (a) Gender-wise distribution of patients, (b) distribution of NCC based on the age group

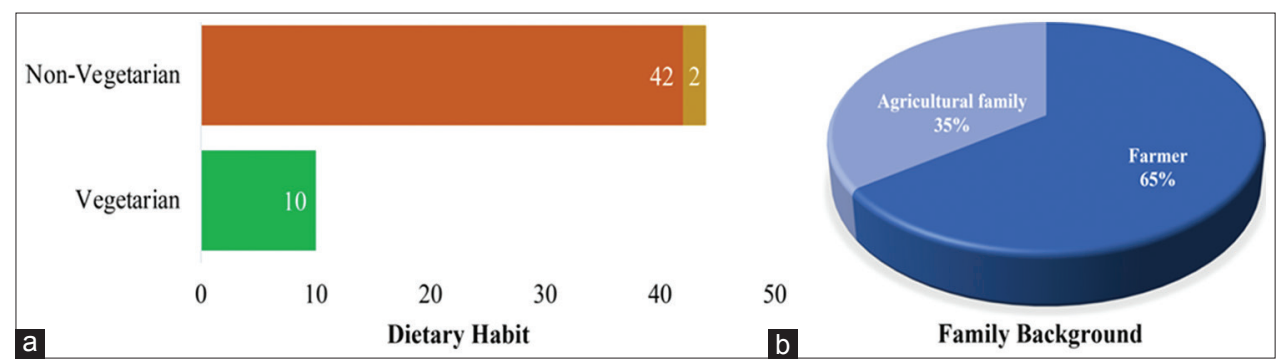

Figure 3: (a-b) Representative graph showing the relationship between dietary habits and NCC

were general meat consumers (GMC). Together, our data showed that NCC is highly prevalent in non-vegetarian compared to vegetarian. But, NCC is more common in GMC rather than PE among non-vegetarians. In addition, socioeconomic background of NCC patients $(n=54)$, in which $65 \%(n=35)$ were farmers and $35 \%(n=19)$ belongs to family with agricultural background as shown in Figure $3 \mathrm{~b}$.

\section{Treatment, Prognosis, and Follow-up}

All patients with an episode of seizure were treated with a single anti-epileptic drug and had a better prognosis. But, among 142 cases, two seizure patients without NCC were reported to be dead due to sepsis and hemorrhagic stroke. While most patients had intraparenchymal involvement, one case had cyst (non-communicating hydrocephalus) in subarachnoid space but, the patient was asymptomatic. This patient refused for the option of surgery and currently under antiepileptic drugs. Currently, all patients are living a healthy life without a seizure.

\section{DISCUSSION}

NCC is a much common socioeconomic, public health and agricultural problems of LMICs, including Nepal. T. solium acknowledges poor-sanitary habit, poverty and underdevelopment for the causes of NCC, in which seizures are the common manifestation in developing countries. ${ }^{8}$ Interestingly, seizures and epilepsy has been interpreted synonymous but, clear distinctions between them have been overlooked based on distinctive characteristics that facilitate accurate diagnosis for effective treatment. ${ }^{9}$ In this study, we presented the high prevalence of NCC (38\%) in patients with seizures. Further, our data revealed that eating pork meat might not be linked directly with the cause of NCC. Though, pork is intermediate host for the larva of T. solium it doesn't determine eating pork meat cause NCC. Also, our data support the reason which showed only few patients $(n=2)$ with habit of eating pork meat that is significantly lower compared to the vegetarian group $(n=10)$ consistent with previous report. ${ }^{10}$ Furthermore, $95 \%$ of Indian patients with NCC were vegetarians and less than 1-2\% were pork eaters or never had pork meat diet. ${ }^{11}$ Further, the prevalence of NCC was distributed based on the age group between 10-80 years. The distribution showed higher proportion of NCC in age group between 1020 years and lower proportion in 30-40 years. Similar predominance of NCC in 10-20 years was reported. ${ }^{12}$ Surprisingly, few other data reported significantly great number of NCC patients were 20-30 years old that was contrast from our observation. ${ }^{10,13}$ Furthermore, our data showed higher number of male patients with NCC that is consistent with the previous report. ${ }^{5,6}$ All patients disclosed the eating habit of raw vegetables directly from the agricultural field and even without washing that 
could be the reason for cause of NCC in the patients of COMS-TH.

Our study demonstrates that NCC is the most common cause of seizure in patients visiting COMS-TH and the association of NCC with pork eaters are lower than vegetarians. This data is not enough to conclude the true prevalence of Nepal. But medical practitioners and health authorities should take effort to spread awareness on standard personal hygiene and transmission risk of NCC to minimize the number of NCC. Also, it is not logical to correlate such predominance based on demographic profile. Several factors, such as total number of patients, personal hygiene, poverty level of patients, endemic area, pig husbandry background, influences the retrospective analysis of one particular place. It is highly recommended to perform systemic meta-analysis of data available from all the hospital of the country that will help to determine the true prevalence and understand the epidemiology of NCC.

\section{PRESPECTIVES}

\section{Preventable but neglected disease}

Neurocysticercosis is the most frequent and preventable cause of acquired epilepsy in developing countries. But neglected due to local customs, customary festivals of eating raw pork, and beliefs and practice of open defecation near river. Such practices ensure continuing transmission of the diseases.

\section{Prevention and control}

Both medical practitioners and health authorities should implement the strategies for improving chemotherapy for NCC in human and vaccination of pigs that will help to eliminate cysticercosis. Several measures listed below ensure the prevention and control of diseases transmission, such as health education and awareness on zoonotic diseases, standard personal hygiene (hand wash before meal), adequate drinking of pure water and proper sanitation, management and surveillance of pig farming communities, standard pig farming practices and strict meat inspection program etc.

\section{CONCLUSION}

Neurocysticercosis is the most common cause of new onset seizure in Nepalese patients visiting COMS-TH, which is more predominant at age of 10-20 years. Further, the common cause of NCC was associated with habit of eating raw vegetables directly from the agricultural field and poor sanitation due to low socioeconomic status. The true prevalence of NCC in Nepal is unavailable and incomplete due to infrequently recognized or outdated data. Our current data showed the high prevalence of NCC in patients with seizure visiting COMS-TH and warrant the need for nationwide analysis to reveal true prevalence of NCC in Nepal.

\section{ACKNOWLEDGEMENT}

We: authors sincerely thank Dr. PVS Rana and Dr. Subish Palaian for guidance and continuous support. Finally, we are thankful to Rupendra Shrestha (Associate Scientist) for reviewing and editing the manuscript.

\section{REFERENCE}

1. Gripper LB and Welburn SC. Neurocysticercosis infection and disease-A review. Acta Trop 2017; 166:218-224.

2. Del Brutto OH. Neurocysticercosis: A review. ScientificWorldJournal 2012; 2012:159821.

3. Fabiani $S$ and Bruschi F. Neurocysticercosis in Europe: Still a public health concern not only for imported cases. Acta Trop 2013; 128(1):18-26.

4. Crompton DWT, Savioli L, Daumerie D and World Health Organization/Department of Control of Neglected Tropical Diseases. Working to overcome the global impact of neglected tropical diseases: First WHO report on neglected tropical diseases. World Health Organization; 2010. p. 140.

5. Ojha R, Shah D, Shrestha A, Koirala S, Dahal A, Adhikari K, et al. Neurocysticercosis in Nepal: A retrospective clinical analysis. Neuroimmunol Neuroinflammation 2015; 2(3):167.

6. Rao KS, Adhikari S, Gauchan E, Sathian B, BKG, Basnet S, et al. Time trend of neurocysticercosis in children with seizures in a tertiary hospital of western Nepal. PLoS Negl Trop Dis 2017; 11(5):e0005605.

7. Gupta MM, Chaudhary N, Pathak S, Agrawal N, Yadav J, Shrestha S, et al. Neurocysticercosis in children with seizures: A cross-sectional study. Int J Pediatr 2018; 2018:1030878.

8. Pal D, Carpio A and Sander J. Neurocysticercosis and epilepsy in developing countries. J Neurol Neurosurg Psychiatry 2000; 68(2):137.

9. Carpio $\mathrm{A}$ and Romo ML. The relationship between neurocysticercosis and epilepsy: An endless debate. Arq Neuropsiquiatr 2014; 72(5):383-390.

10. Prudhivi S, Meeniga S, Toleti S, Myneni RB and Rao S. Prevalence of Neurocysticercosis in and around Chinakakani, Andhra Pradesh, South India. Int J Curr Mircobiology Appl Sci 2015; 4(10):424-431.

11. Rajshekhar $V$, Joshi DD, Doanh $N Q$, van De $N$ and Xiaonong Z. Taenia solium taeniosis/cysticercosis in Asia: Epidemiology, impact and issues. Acta Trop 2003; 87(1):53-60.

12. Kumar A, Khan SA, Khan S, Das S, Anurag and Negi KS. A study of Neurocysticercosis in the foothills of the Himalayas. Int J Infect Dis 2006; 10(1):79-82.

13. Sailaja $M$ and S.Umadevi. A study on the prevalence of Neurocysticercosis in a tertiary hospital. Int J Res Heal Sci 2014; 2(4):1105-1109. 
Authors Contribution:

NK- Concept, design and data analysis; RS- Data analysis, manuscript preparation, review and editing.

Work attributed to:

Department of Neuromedicine, College of Medical Science and Teaching Hospital, Nepal.

Orcid ID:

Dr. Nisha Khanal- (1) https://orcid.org/0000-0001-8382-5304

Dr. Reena Shrestha- (i) https://orcid.org/0000-0001-8412-282X

Source of Support: None, Conflict of Interest: The authors declare no conflict of interest. 